\title{
Quantitative scanning probe microscope topographies by charge linearization of the vertical actuator
}

\author{
Andrew J. Fleming ${ }^{\text {a) }}$ \\ School of Electrical Engineering and Computer Science, University of Newcastle, \\ Callaghan, New South Wales 2308, Australia
}

(Received 22 February 2010; accepted 18 August 2010; published online 14 October 2010)

\begin{abstract}
Many forms of scanning probe microscopy require a piezoelectric actuator to vary the probe-sample distance. Examples include constant-force atomic force microscopy and constant-current scanning tunneling microscopy. In such modes, the topography of the sample is reconstructed from the voltage applied to the vertical piezoelectric actuator. However, piezoelectric actuators exhibit significant hysteresis which can produce up to $14 \%$ uncertainty in the reproduced topography. In this work, a charge drive is used to linearize the vertical piezoelectric actuator which reduces the error from $14 \%$ to $0.65 \%$. (C) 2010 American Institute of Physics. [doi:10.1063/1.3488359]
\end{abstract}

\section{INTRODUCTION}

Scanning probe microscopes (SPMs) record localized physical interactions between a probe and sample as a function of position. A diverse range of techniques and probes have become available to image properties such as topography, electrical and mechanical forces, chemical bonding, and biological interactions. ${ }^{1-5}$

Many popular modes of scanning probe microscopy require a vertical feedback system to regulate the probe-sample interaction. Examples include constant-current scanning tunneling microscopy and constant-force atomic force microscopy. These techniques ensure that the probe-sample interaction is kept constant by varying the probe or sample height. Rather than recording the cantilever deflection, which is a highly nonlinear function of topography, the image is reproduced from the control voltage applied to the vertical actuator. It is assumed that the control voltage is directly proportional to the position and hence topography.

Due to their high speed, compact size, and essentially infinite resolution, piezoelectric actuators are used almost exclusively in scanning probe microscopes. SPM scanners and vertical positioners are usually constructed from either piezoelectric tube actuators ${ }^{6,7}$ or faster piezoelectric stack actuators. ${ }^{8}$ Although scanners constructed from piezoelectric actuators have extremely high resolution, the overall accuracy is limited by creep and hysteresis. ' For example, the positioning error due to hysteresis in a piezoelectric tube actuator has been reported to be $\pm 9.7 \%$ of the scan range. ${ }^{10}$ This implies a maximum positioning error of almost $20 \%$ between the forward and backward scanning paths.

To avoid imaging artifacts, SPMs require some form of compensation for positioning nonlinearity. Methods to accomplish this, including feedback and feedforward control, have been recently surveyed. ${ }^{9,11,12}$ However, these methods are aimed at reducing lateral positioning error and do not consider vertical axis nonlinearity.

\footnotetext{
${ }^{\text {a)} E l e c t r o n i c ~ m a i l: ~ a n d r e w . f l e m i n g ~ @ ~ N e w c a s t l e . e d u . a u . ~}$
}

It is generally accepted that piezoelectric nonlinearity can be neglected in the vertical axis since hysteresis is voltage dependent and the sample features will be small compared to the full-scale range of the scanner. However, this can be a poor assumption, particularly when the sample substrate is sloping which requires large excursions from the vertical positioner. It is also not uncommon for sample features to exceed $10 \%$ of the full-scale range, especially in microscopes designed for high speed. ${ }^{8,13-17}$ Even at 5\% of the full-scale range, hysteresis has been shown to result in up to $\pm 2 \%$ error and $\pm 4.9 \%$ error at $20 \%$ of the full-scale range. ${ }^{10}$ Thus, it should not be neglected if quantitative topographical information is desired.

Recently, the presence of vertical nonlinearity has been addressed by metrological SPMs. ${ }^{18}$ Rather than simply recording the applied actuator voltage, metrological SPMs contain a position sensor to measure the vertical displacement directly. As the actuator nonlinearity is bypassed, the recorded image is a quantitative reproduction of the sample topography.

The disadvantage of metrological SPMs is inherent in their design; they require a linear position sensor capable of large range, wide bandwidth, and high resolution. This requirement can significantly increase the scanner complexity and dramatically increases the cost. Furthermore, typical position sensors are much noisier than the control voltage applied to a vertical actuator. For example, the peak-to-peak noise of the laser interferometer used in the aforementioned $\mathrm{SPM}$ is $4 \mathrm{~nm}^{18}$

The most recent generation of commercial atomic force microscopes use a capacitive or inductive position sensor to directly measure the vertical topography. These sensors have a range of around $10 \mu \mathrm{m}$, an uncorrected linearity of around $1 \%$, and a typical noise density of $3 \mathrm{pm} / \sqrt{\mathrm{Hz}}$. If the noise is assumed to be Gaussian distributed with a bandlimited constant spectral density, the peak- to-peak noise is approximately 


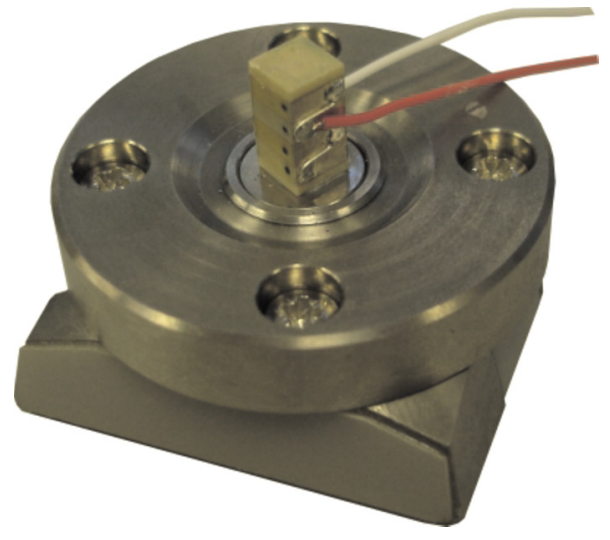

FIG. 1. (Color online) The vertical positioner is a $10 \mathrm{~mm}$ piezoelectric stack actuator (Noliac SCMAP07) bonded to a standard base plate. The sample holder is affixed to the top of the stack.

peak-to-peak noise $=6 \times$ noise density

$$
\times \sqrt{2 \times \text { bandwidth. }} \text {. }
$$

Hence, a typical position sensor with a bandwidth of $1 \mathrm{kHz}$ exhibits a peak-to-peak noise of approximately $800 \mathrm{pm}$. This is insufficient for atomic resolution; however, if the bandwidth is reduced to $10 \mathrm{~Hz}$, the noise reduces to $80 \mathrm{pm}$ and atomic resolution can be obtained.

A penalty of reducing the position sensor bandwidth is lower imaging speed. Based on the assumptions of triangular scanning and ten features per line, the position sensor bandwidth needs to be approximately 200 times the scan-rate for "sharp" samples and 20 times the scan-rate for "smooth" samples. Hence, if the position sensor bandwidth is limited to $10 \mathrm{~Hz}$, the scan-rate must be limited to $0.5 \mathrm{~Hz}$ for smooth samples and $0.05 \mathrm{~Hz}$ for sharp samples. A scan-rate of $0.05 \mathrm{~Hz}$ would require $8 \mathrm{~min}$ for a $100 \times 100$ resolution image, which is prohibitively slow.

Due to the noise generated by position sensors, they are rarely used in high resolution imaging. Instead, the topography is acquired in the traditional manner from the applied actuator voltage, albeit at the expense of poor linearity as discussed above. To appreciate the improvement in noise performance, consider a standard $200 \mathrm{~V}$ amplifier with a $1 \mathrm{kHz}$ bandwidth and a peak-to-peak noise of $1 \mathrm{mV}$ (e.g., the PiezoDrive PDL200 amplifier). Using a piezoelectric actuator with a range of $10 \mu \mathrm{m}$, the sensitivity is $50 \mathrm{~nm} / \mathrm{V}$ which implies a peak-to-peak noise of only $50 \mathrm{pm}$. This figure is only $6 \%$ of the position sensor noise and demonstrates why the applied actuator voltage is preferred for atomic scale imaging.

In this work, a new approach is demonstrated for obtaining quantitative topographical information from a standard SPM. Rather than the use of a position sensor or hysteresis model, the vertical axis voltage amplifier is simply replaced by a charge drive. As piezoelectric actuators respond more linearly to charge or current rather than voltage, ${ }^{19}$ hysteresis is substantially reduced. In Sec. III, the hysteresis of a piezoelectric stack actuator is reduced from $14.3 \%$ of the full-scale range to $0.65 \%$. In Sec. IV, this reduction is demonstrated to dramatically improve the linearity and reproducibility of the recorded topography. This technique can be easily retrofitted

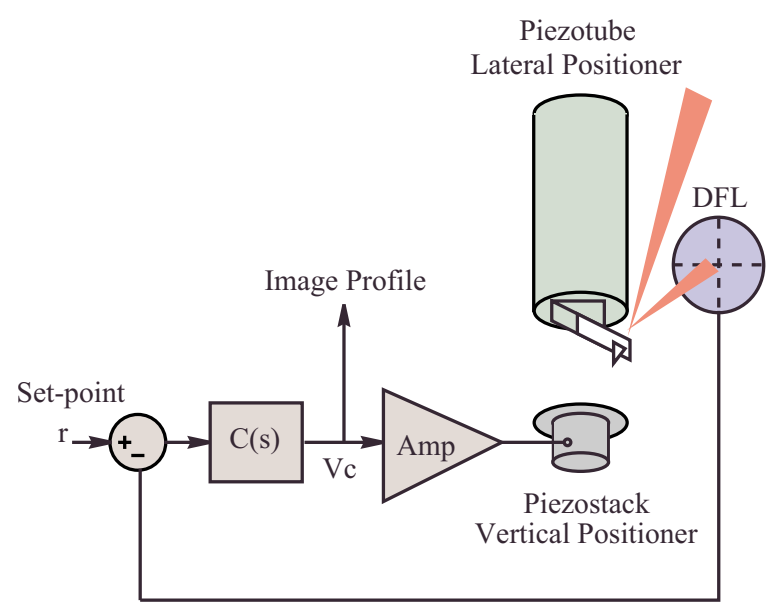

FIG. 2. (Color online) Schematic diagram of the vertical feedback loop operating in constant- force contact-mode AFM. The image profile is the voltage applied to the amplifier.

to any commercial SPM and avoids the problem of actuator nonlinearity without the addition of position sensor noise.

This paper proceeds in the following section with a description of the experimental setup.

\section{EXPERIMENTAL SETUP}

The proposed techniques are demonstrated on an NT-MDT Ntegra SPM arranged in a scan-by-probe configuration. The scanner is an NT-MDT Sm8122cl piezoelectric tube scanner with $100 \mu \mathrm{m}$ lateral range and $10 \mu \mathrm{m}$ vertical range. Since the scanner resonance frequency is only $680 \mathrm{~Hz},{ }^{20}$ the vertical positioning function is replaced by a high-speed piezoelectric stack actuator as pictured in Fig. 1. The actuator is a $10 \mathrm{~mm}$ long Noliac SCMAP07 stack actuator, epoxy-bonded to a standard microscope base. The sample holder is glued directly onto the top of the actuator. A full-scale extension of $10.5 \mu \mathrm{m}$ is developed from a $200 \mathrm{~V}$ applied voltage.

The use of a separate vertical positioning stage eliminates the presence of low-frequency lateral resonance modes in the vertical feedback loop. This approach has been reported to increase the $\mathrm{z}$-axis bandwidth by more than an order of magnitude. . $^{17,20,21}$

The maximum vertical feedback bandwidth has previously been shown to be ${ }^{17}$

$$
\text { maximum bandwidth }=\frac{\text { resonance frequency }}{\text { peak amplitude }},
$$

where the peak amplitude is the magnitude at the resonance frequency divided by the dc gain.

The first resonance frequency of the vertical stage pictured in Fig. 1 occurs at $20.3 \mathrm{kHz}$ and the peak amplitude is 2.6 (or $8.3 \mathrm{~dB}$ ). This allows a maximum vertical feedback bandwidth of $7.8 \mathrm{kHz}$, which is 65 times faster than the standard maximum bandwidth of $120 \mathrm{~Hz} .^{17}$

The operation of the vertical feedback loop during constant-force contact-mode AFM is illustrated in Fig. 2. The controller $C(s)$ maintains a constant probe-sample interaction while the image profile is obtained from the voltage applied to the vertical axis amplifier. This mode of operation is simi- 
A. Full Range Piezoelectric Hysteresis

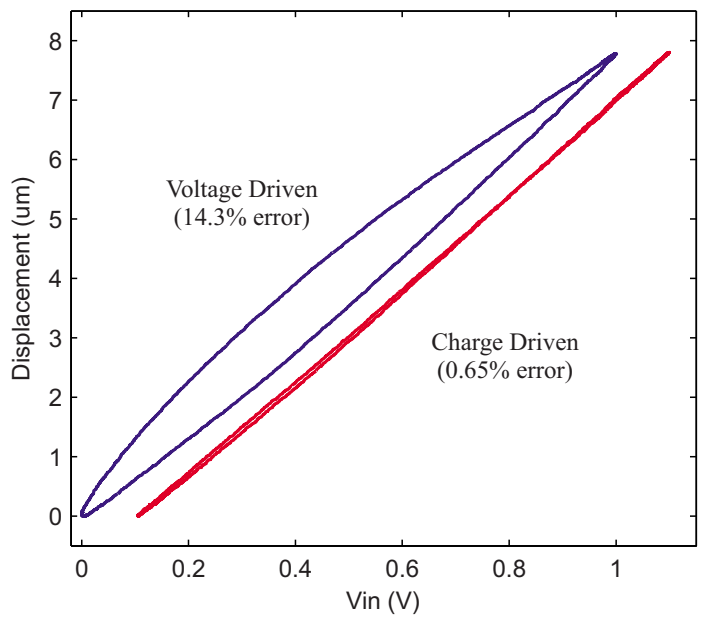

B. $15 \%$ Range Piezoelectric Hysteresis

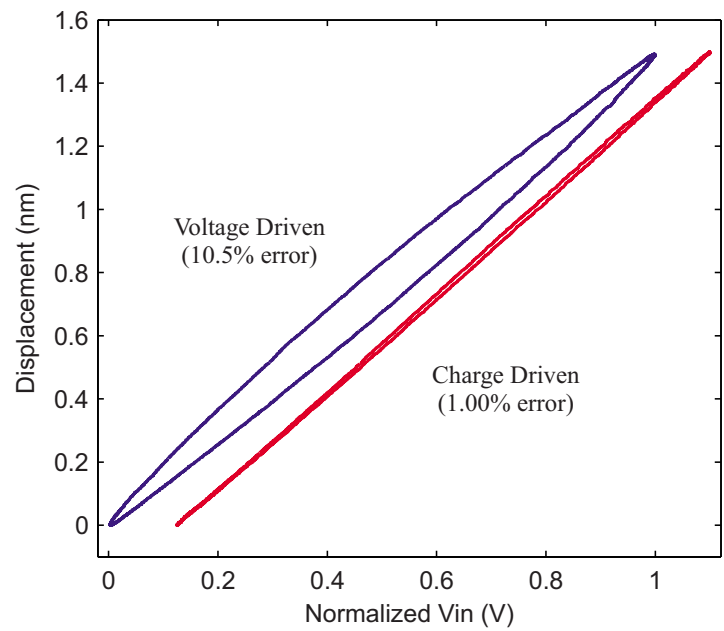

FIG. 3. (Color online) A comparison of hysteresis exhibited by the voltagedriven and charge-driven piezoelectric positioner shown in Fig. 1. The input signal was a $100 \mathrm{~Hz}$ sine wave with a peak-to-peak voltage of $150 \mathrm{~V}$ in (a) and $30 \mathrm{~V}$ in (b). (The charge-driven results are offset for clarity).

lar to many forms of SPM where the probe-sample interaction is controlled. Different operating modes use different feedback variables. For example, in constant-force contactmode AFM, the feedback variable is cantilever deflection. In constant-current STM, the feedback variable is the tunneling current. Other feedback variables include the cantilever oscillation magnitude in tapping-mode AFM and the fiber oscillation magnitude in scanning near-field optical microscopy.

The linearity of the vertical axis positioner is examined in Sec. III.

\section{LINEARIZATION OF THE VERTICAL AXIS}

In Fig. 2, the vertical feedback loop of an AFM is shown while operating in constant-force contact-mode. To derive the image profile from the control voltage $V_{c}$, the sensitivity of the amplifier $K_{a}$ and positioner $K_{p}$ must be known. For the setup described above, the amplifier gain is $K_{a}=20 \mathrm{~V} / \mathrm{V}$ and the positioner sensitivity is $K_{p}=53 \mathrm{~nm} / \mathrm{V}$. The image profile is thus $V_{c}(x, y) \times K_{a} K_{p}$ or $V_{c}(x, y) \times 1.06 \mu \mathrm{m}$.

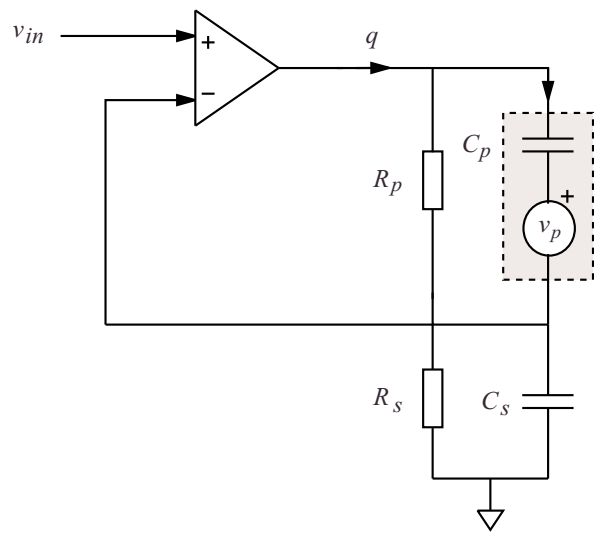

FIG. 4. (Color online) Simplified schematic diagram of a charge drive. The piezoelectric load is shaded in gray.

Clearly the image profile relies on a proportional relationship between the applied voltage and resulting displacement. The validity of this assumption was tested by applying a $10 \mathrm{~Hz}, 150 \mathrm{~V}$ sine wave to the actuator, then recording the displacement with a Polytec-PI MSV400 laser vibrometer. The results are plotted in Fig. 3(a). The maximum difference in position between the two points with the same applied voltage was $1.1 \mu \mathrm{m}$ or $14.3 \%$ of the range. A second experiment was conducted to examine the nonlinearity when operating at only $30 \mathrm{~V}$ or $15 \%$ of the full-scale range. Although reduced, the error due to hysteresis was still $157 \mathrm{~nm}$ or $10.5 \%$ of the range. From these results, it can be concluded that a quantitative topography cannot be obtained directly from the control voltage $V_{c}$. Similar magnitudes of nonlinearity have been reported using piezoelectric tubes rather than stack actuators. ${ }^{10}$

As discussed in Sec. I, metrological SPMs use a position sensor to bypass the actuator nonlinearity. In the present work, rather than bypassing the actuator, the actuator is linearized by applying charge instead of voltage.

It has been known since the 1980s that piezoelectric transducers respond more linearly to current or charge rather than voltage. ${ }^{19}$ However, practical problems with drift and the floating nature of the load were only recently solved. ${ }^{22,23}$ Since then, charge drives have been demonstrated to reduce the hysteresis of SPM tube scanners by up to $93 \% .{ }^{10}$ This corresponds to a maximum nonlinearity of less than $1 \%$ that effectively eliminates the need for feedback or feedforward control in dynamic applications.

A simplified schematic diagram of the charge drive used in this work is shown in Fig. 4. Since the piezoelectric stack actuator does not require a grounded electrode, the floating-

TABLE I. The maximum error due to hysteresis of the voltage-driven and charge-driven piezoelectric actuator.

\begin{tabular}{lcccccc}
\hline \hline & \multicolumn{2}{c}{ Absolute error } & & \multicolumn{2}{c}{$\%$ error } & \\
\cline { 2 - 3 } $\begin{array}{l}\text { Range } \\
(\mu \mathrm{m})\end{array}$ & Voltage & $\begin{array}{c}\text { Charge } \\
(\mathrm{nm})\end{array}$ & & $\begin{array}{c}\text { Voltage } \\
(\%)\end{array}$ & $\begin{array}{c}\text { Charge } \\
(\%)\end{array}$ & $\begin{array}{c}\text { Reduction } \\
(\%)\end{array}$ \\
\hline 7.8 & $1.1 \mu \mathrm{m}$ & 51 & & 14.3 & 0.65 & 95 \\
1.5 & $157 \mathrm{~nm}$ & 15 & & 10.5 & 1.00 & 90 \\
\hline \hline
\end{tabular}




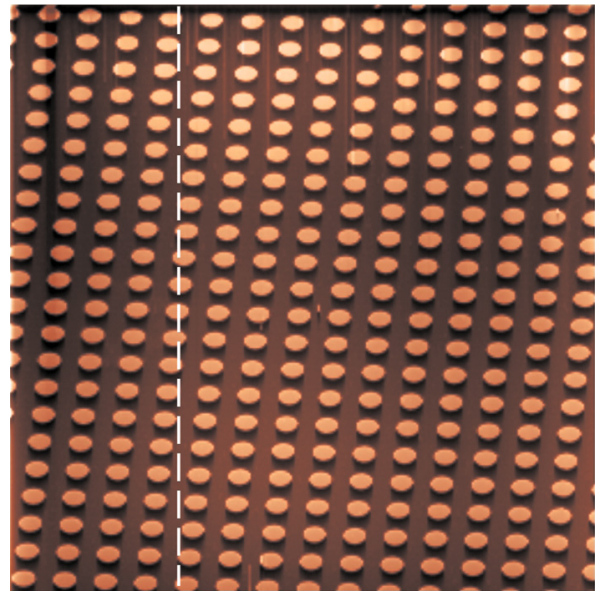

FIG. 5. (Color online) The topography of a BudgetSensors HS-100MG calibration grating imaged in constant-force contact-mode. The scan area is $100 \mu \mathrm{m}$ and the feature height is $100 \mathrm{~nm}$. The dashed line indicates the location of the single profile line plotted in Fig. 6.

load circuit $^{22}$ was used instead of the grounded-load arrangement. ${ }^{23}$ In Fig. 4, the piezoelectric load is modeled as a capacitor $C_{p}$ and voltage source $v_{p}$. The high-gain feedback loop works to equate the applied reference voltage $v_{\text {in }}$ to the voltage across a sensing capacitor $C_{s}$. Neglecting the resistances $R_{p}$ and $R_{s}$, the charge $q$ is

$$
q=V_{i n} C_{s}
$$

That is, the gain is $C_{s}$ Coulombs/volt. When connected to a capacitive load, the equivalent voltage gain is $C_{s} / C_{p}$. As discussed previously, ${ }^{24}$ the existence of $R_{p}$ and $R_{s}$ introduces error at low frequencies. However, by ensuring that the ratio of resistances is equal to the inverse ratio of capacitances, low-frequency error can be eliminated. That is, by setting

$$
\frac{R_{p}}{R_{s}}=\frac{C_{s}}{C_{p}},
$$

the amplifier has a constant gain of $C_{s}$ Coulombs/volt over all frequencies.

As the actuator capacitance is $C_{p}=330 \mathrm{nF}$, a sensing capacitance of $22 \mu \mathrm{F}$ was chosen to provide a voltage gain of 66. To maintain this voltage gain at dc, the parallel resistances were chosen to be $R_{p}=6.6 \mathrm{M} \Omega$ and $R_{s}=100 \mathrm{k} \Omega$. This circuit was implemented by a PiezoDrive PDQ200 charge amplifier.

The response of the charge-driven piezoelectric actuator is plotted in Fig. 3. The maximum nonlinearity with a $150 \mathrm{~V}$ excitation is $51 \mathrm{~nm}$ or $0.65 \%$ of the range. With a $30 \mathrm{~V}$ excitation, the maximum nonlinearity is $15 \mathrm{~nm}$ or $1 \%$ of the range. These results are summarized in Table I. Although the maximum residual hysteresis of $1 \%$ is not comparable to the linearity of a laser interferometer, this magnitude of error is sufficient for many applications that require quantitative topographical information.

In addition to the improvement in linearity, charge drives have a number of advantages over physical position sensors. First, they are low-cost and are easily retrofitted to any SPM without mechanical modifications. Second, the random noise

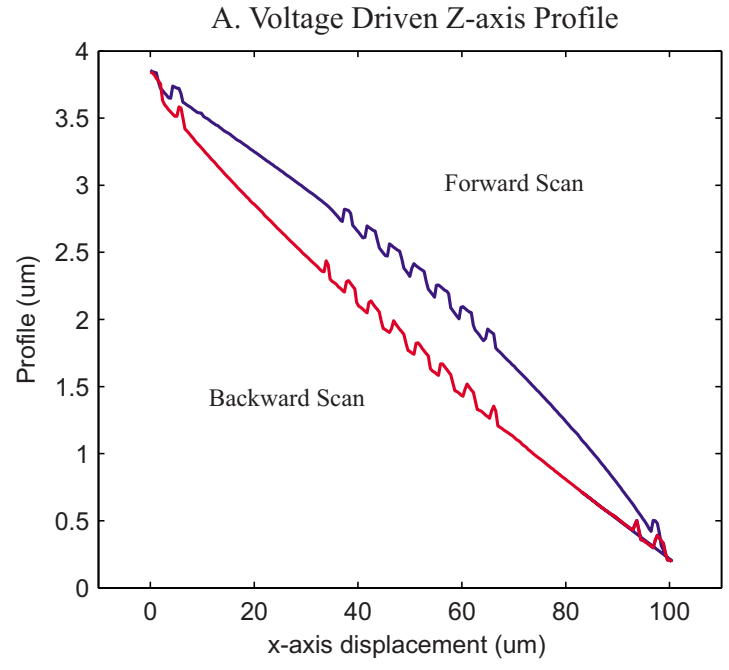

B. Charge Driven Z-axis Profile

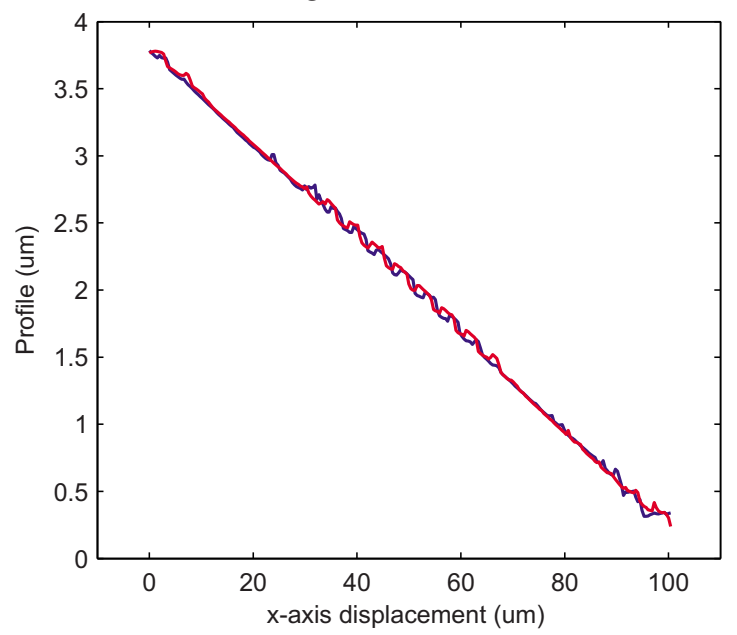

FIG. 6. (Color online) The profile of a single image line acquired using a (a) voltage-driven and (b) charge-driven vertical positioner.

produced by a charge drive is similar to that of a voltage amplifier, ${ }^{10}$ which, as discussed in Sec. I, is significantly less than a physical position sensor.

The random noise generated by a charge drive is similar to a voltage amplifier since the topology of both circuits is almost identical. In both cases, the amplifier's input noise voltage is the dominant process since this is multiplied by the gain of the circuit. Since charge amplifiers have a highimpedance output, they are more susceptible to interference than voltage amplifiers. If the output is not appropriately shielded, additional noise can result.

With the load capacitance attached, the output noise voltage of the PDQ200 charge amplifier was measured to be $1.5 \mathrm{mV}$ rms. This was measured by a Fluke 289 multimeter with a $100 \mathrm{kHz}$ measurement bandwidth. The same circuit configured as a voltage amplifier generated an output voltage noise of $1.2 \mathrm{mV} \mathrm{rms}$, hence there is little noise penalty when using a charge drive.

Another consideration with charge amplifiers is the bandwidth. As the circuit topology of a charge amplifier is similar to a voltage amplifier, the bandwidth is also similar. However, for effective rejection of the disturbance caused by 
hysteresis, the internal loop gain needs to be greater than approximately $20 \mathrm{~dB}$. This occurs at frequencies lesser than one-tenth of the bandwidth.

The bandwidth of the PDQ200 charge amplifier was measured to be $51 \mathrm{kHz}$. This means that significant hysteresis rejection only occurs at frequencies below $5 \mathrm{kHz}$. This is not a significant disadvantage as all amplifiers have a bandwidth of at least ten times the maximum signal frequency so that phase lag and magnitude shift can be avoided. In this case, it is not possible to experimentally measure the hysteresis response over a wide frequency range due to the current limit of the amplifier and self-heating of the actuator.

\section{IMAGING PERFORMANCE}

In this section we compare the topographic profiles of an AFM image acquired using a voltage-driven and chargedriven vertical actuator. The sample under consideration is a BudgetSensors HS-100MG calibration grating. A constantforce contact-mode AFM image of the sample (using a voltage amplifier) is shown in Fig. 5. The sample slope was removed by subtracting a second-order plane.

Also shown in Fig. 5 is a dashed line that illustrates the location of a single profile line. When using a voltage-driven vertical actuator, the raw profile is plotted in Fig. 6(a). In this plot, the piezoelectric hysteresis is observed to add significant curvature to the profile and introduce a large discrepancy between the forward and backward scan paths. After replacing the voltage amplifier with a charge drive, the same image line is plotted in Fig. 6(b). With the hysteresis reduced to a negligible level, the linearity and reproducibility are greatly improved.

\section{CONCLUSIONS}

In this work, piezoelectric hysteresis was demonstrated to cause an uncertainty of up to $14.3 \%$ in the topography of an SPM image. Even small vertical excursions resulted in a $10.5 \%$ error due to hysteresis. This magnitude of error precludes the acquisition of quantitative topographical information from an SPM without a position sensor or other compensation for vertical nonlinearity.

To reduce the image uncertainties, a charge drive was proposed to linearize the vertical piezoelectric actuator. In experiments, the error due to hysteresis was reduced by at least $90 \%$ to $1 \%$ of the range. This was sufficient to eliminate visible artifacts in a constant-force contact-mode AFM profile.

Although displacement sensors such as laser interferometers can provide better linearity than a charge-driven piezo- electric actuator, they also require significant mechanical modifications, are costly, and may be too noisy to achieve atomic resolution. Charge amplifiers are a simple, highperformance alternative for conventional SPMs when topographical uncertainties of $1 \%$ can be tolerated.

\section{ACKNOWLEDGMENTS}

This research was supported by an Australian Research Council Discovery Project (Grant No. DP0986319). Experiments were carried out at the Laboratory for Dynamics and Control of Nanosystems at the University of Newcastle.

${ }^{1}$ M. J. Brukman and D. A. Bonnell, Phys. Today 61(6), 36 (2008).

${ }^{2}$ Y. F. Dufrêne, Nat. Rev. Microbiol. 6, 674 (2008).

${ }^{3}$ Scanning Probe Microscopy and Spectroscopy. Theory, Techniques, and Applications, 2nd ed., edited by D. Bonnell (Wiley-VCH, Hoboken, 2001).

${ }^{4}$ E. Meyer, H. J. Hug, and R. Bennewitz, Scanning Probe Microscopy. The Lab on a Tip (Springer-Verlag, Heidelberg, 2004).

${ }^{5}$ The Handbook of Nanotechnology, edited by B. Bhushan (SpringerVerlag, Berlin, 2004).

${ }^{6}$ G. Binnig and D. P. E. Smith, Rev. Sci. Instrum. 57, 1688 (1986).

${ }^{7}$ S. O. R. Moheimani, Rev. Sci. Instrum. 79, 071101 (2008).

${ }^{8}$ G. E. Fantner, G. Schitter, J. H. Kindt, T. Ivanov, K. Ivanova, R. Patel, N. Holten-Andersen, J. Adams, P. J. Thurner, I. W. Rangelow, and P. K. Hansma, Ultramicroscopy 106, 881 (2006).

${ }^{9}$ S. Devasia, E. Eleftheriou, and S. O. R. Moheimani, IEEE Trans. Control Syst. Technol. 15, 802 (2007).

${ }^{10}$ A. J. Fleming and K. K. Leang, Ultramicroscopy 108, 1551 (2008).

${ }^{11}$ J. A. Butterworth, L. Y. Pao, and D. Y. Abramovitch, Asian J. Control 11, 175 (2009).

${ }^{12}$ G. M. Clayton, S. Tien, K. K. Leang, Q. Zou, and S. Devasia, J. Dyn. Syst. Meas. 131, 061101 (2009).

${ }^{13}$ T. Ando, N. Kodera, T. Uchihashi, A. Miyagi, R. Nakakita, H. Yamashita, and K. Matada, e-J. Surf. Sci. Nanotechnol. 3, 384 (2005).

${ }^{14}$ A. D. L. Humphris, M. J. Miles, and J. K. Hobbs, Appl. Phys. Lett. 86, 034106 (2005).

${ }^{15}$ M. J. Rost, L. Crama, P. Schakel, E. van Tol, G. B. E. M. van VelzenWilliams, C. F. Overgauw, H. ter Horst, H. Dekker, B. Okhuijsen, M. Seynen, A. Vijftigschild, P. Han, A. J. Katan, K. Schoots, R. Schumm, W. van Loo, T. H. Oosterkamp, and J. W. M. Frenken, Rev. Sci. Instrum. 76, 053710 (2005).

${ }^{16}$ L. M. Picco, L. Bozec, A. Ulcinas, D. J. Engledew, M. Antognozzi, M. Horton, and M. J. Miles, Nanotechnology 18, 044030 (2007).

${ }^{17}$ A. J. Fleming, B. J. Kenton, and K. K. Leang, Ultramicroscopy 110, 1205 (2010).

${ }^{18}$ R. Merry, M. Uyanik, R. van de Molengraft, R. Koops, M. van Veghel, and M. Steinbuch, Asian J. Control 11, 130 (2009).

${ }^{19}$ C. V. Newcomb and I. Flinn, Electron. Lett. 18, $442(1982)$

${ }^{20}$ A. J. Fleming, IEEE Trans. Control Syst. Technol. (to be published).

${ }^{21}$ G. Schitter, Tech. Mess. 76, 266 (2009).

${ }^{22}$ K. A. Yi and R. J. Veillette, IEEE Trans. Control Syst. Technol. 13, 517 (2005).

${ }^{23}$ A. J. Fleming and S. O. R. Moheimani, Rev. Sci. Instrum. 76, 073707 (2005).

${ }^{24}$ A. J. Fleming and S. O. R. Moheimani, IEEE Trans. Control Syst. Technol. 14, 33 (2006). 